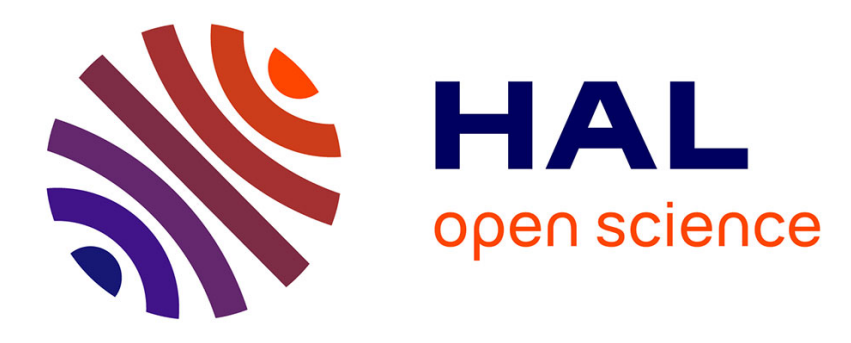

\title{
Reticular plasmon resonance detection properties of metal nanoparticles
}

\author{
Mohamed El Barghouti, Abdellatif Akjouj, Abdellah Mir
}

\section{To cite this version:}

Mohamed El Barghouti, Abdellatif Akjouj, Abdellah Mir. Reticular plasmon resonance detection properties of metal nanoparticles. Physica E: Low-dimensional Systems and Nanostructures, 2019, 110, pp.107-114. 10.1016/j.physe.2019.02.010 . hal-03140650

\section{HAL Id: hal-03140650 \\ https://hal.science/hal-03140650}

Submitted on 23 Aug 2021

HAL is a multi-disciplinary open access archive for the deposit and dissemination of scientific research documents, whether they are published or not. The documents may come from teaching and research institutions in France or abroad, or from public or private research centers.
L'archive ouverte pluridisciplinaire HAL, est destinée au dépôt et à la diffusion de documents scientifiques de niveau recherche, publiés ou non, émanant des établissements d'enseignement et de recherche français ou étrangers, des laboratoires publics ou privés. 


\title{
Reticular plasmon resonance detection properties of metal nanoparticles
}

\author{
Mohamed El barghouti ${ }^{\mathrm{a}, *}$, Abdellatif Akjouj ${ }^{\mathrm{b}}$, Abdellah Mir ${ }^{\mathrm{a}}$ \\ ${ }^{a}$ Laboratory of Advanced Materials Studies and Applications (LEM2A), Physics Department, Faculty of Science, Moulay Ismail University, B.P. 11201 Zitoune, Meknès, \\ Morocco \\ ${ }^{\mathrm{b}}$ Institute of Electronics, Microelectronics and Nanotechnology, UMR CNRS 8520, Lille University, FST, Department of Physics, 59655 Villeneuve d'Ascq, France
}

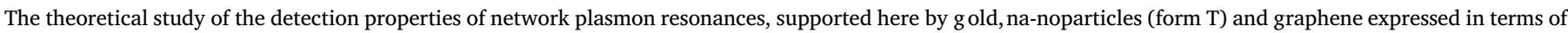

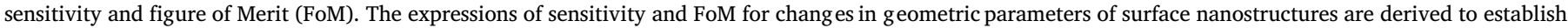

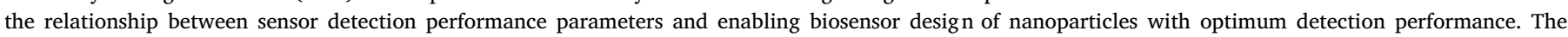

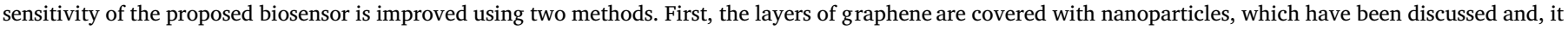

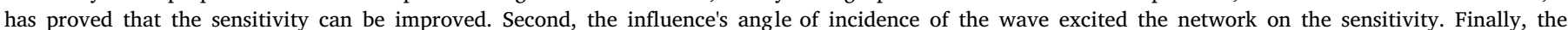

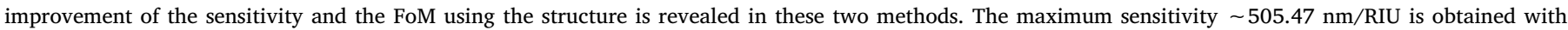
coating of graphene layers and angle of incidence oblique to the surface.
\end{abstract}

\section{Introduction}

It has been known for many years that diffraction can be used for the excitation of surface plasmon polaritons, where as diffraction in regular networks of metal nanoparticles offers the potential to manipulate the properties of localized surface plasmons [1-5]. Surface plasmons (SP) refer to planar waves that propagate along the metal surface which are generated by the interaction between the magnetic transverse polarity (TM) light and the free electrons in the metal surface. When the wave vector of an incident light corresponds to the wave vector of a surface plasmon wave (SPW), the resonant incident light pairs with the surface plasmon and a huge electric field improvement can be achieved, which is called the localized surface plasmon resonance (LSPR) [6,7]. Localized surface plasmon (LSP) can lead to giant improvements in local electromagnetic fields around nanoparticles $[8,9]$ in nanoparticle networks, interactions between individual dipole plasmons can still increase the field improvement and sensor detection performance parameters according to the network settings. Understanding their properties is significantly more complex when metal nanoparticles are deposited on a glass substrate [10-12] or when they interact [13-15]. It is quite clear from the theoretical and experimental studies already available (on the gold nanoparticles) that several parameters govern their behavior [16,17]. But for biosensing applications, gold is not well adapted due to its poor biomolecules adsorption, which limits the sensitivity of the biosensor [18]. So the gold nanoparticles (AuNPs) are used instead of gold layer. Gold nanoparticles have a unique property called localized surface plasmon (LSPR). The AuNPs-based LSPR sensor is widely used for the detection of proteins, antibodies and nucleic acids [19]. In this work it is used for the detection of the incidence light. However, in order to improve sensitivity and merit factor (FoM), there are several schemes to improve the absorption of biosensor, for example, the growth of an affinity layer [20], using gold nanoparticles of well-structured form, and two-dimensional (2D) nano-layers of graphene on AuNPs [18,21].

From an optical point of view, the high refractive index of graphene should induce a redshift of plasmonic resonance when placed above AuNPs [5]. However, an unexpected blue shift in localized plasmon resonance of AuNPs was measured [22,23]. The blue shift origin could not be fully understood and was not reproduced by numerical simulations, which led us to believe that this was due to a charge transfer effect [24,25].

Graphene has aroused considerable interest in recent years because of its distinctive electrical and optical properties [26,27], a highly sensitive LSPR biosensor using the graphene layer to improve the sensitivity caused by the light absorbed by the graphene [28]. They found that sensitivity can be enhanced when the number of layers of graphene increases. In this paper, we theoretically explore the refractive index of several plasmonic models. Typically, a simple factor of merit (FoM),

\footnotetext{
* Corresponding author.

E-mail address: Mohamed.elbarghouti@gmail.com (M. El barghouti).
} 
defined as the sensitivity ratio (resonance wavelength shift in $\mathrm{nm}$ by changing the refractive index of the detection medium $\mathrm{n}_{2}=1$ and 1.333) to the full width at mid-height (FWHM) of the resonance peak is used to compare detection performance $[29,30]$. We note that in biomolecular sensing applications, performance does not depend on the change in the refractive index of the detection medium but on the nanostructure parameters. Therefore, instead of using the refractive index to compare the sensitivity of nanostructure, a layer of graphene (dthickness) is used in the proposed configuration: the graphene layer is utilized to improve the sensitivity and acts as the biomolecular recognition element due to its absorption stability of biomolecules with cyclic carbon-based structures. Here, we observe that when the graphene layer is considered instead of the refractive index, the properly designed localized plasmon resonance sensors exhibit greater sensitivity compared to a structure without graphene $248.82 \mathrm{~nm} / \mathrm{RIU}$. Therefore the change of the angle of the incident wave with the layers of graphene have an axial role in improving the sensitivity. A higher sensitivity is obtained $505.47 \mathrm{~nm} / \mathrm{RIU}$ previously; several plasmonic resonators manufactured on planar surfaces were considered plasmonic interfaces for LSPR detection [31,32]. We also consider plasmonic structures on planar surfaces that can be manufactured by ascending deposit methods. In particular, we study the structure shown in Fig. 1. For simulations, the AuNPs are deposited on the largest surface of a substrate consisting of a semi-infinite space in Indium Tin Oxide (ITO) refractive index $n_{1}=2$, with a thin layer of graphene $\left(n_{g}\right)$ deposited on top of gold nanoparticles (form $\mathrm{T}$ ), in order to take the configuration on the graphene a non-absorbent refractive index dielectric $\left(\mathrm{n}_{2}\right)$. The structure is illuminated from the ITO substrate by a linearly polarized planar wave of the incident wave vector $\left(\mathbf{k}_{\text {inc }}\right)$, the direction of which depends on the azimuth angle $\theta$ (between $\mathbf{k}_{\text {inc }}$ and the axis $(\mathrm{Oz})$ ). The dielectric constant for gold, $\varepsilon(\omega)$, is obtained according to the frequency. The graphene is considered a very promising new type of materials for possible applications in several domains (including bioplasmon detection). A 2D nanomaterial that entered the image very recently [33], and came into the picture because of their extraordinary absorption properties [34,35]. Otherwise indicated that the load of mobility of the graphene carriers is as high as $106 \mathrm{~cm}^{2} \mathrm{~V}^{-1} \mathrm{~s}^{-1}$ [36]. Specifically, we will examine the effect of progressively varying the graphene thickness from 0 to $5 \mathrm{~nm}$ (from 0 to 15 layers). Basically, we study numerically the nanostructure of how the layers of graphene are

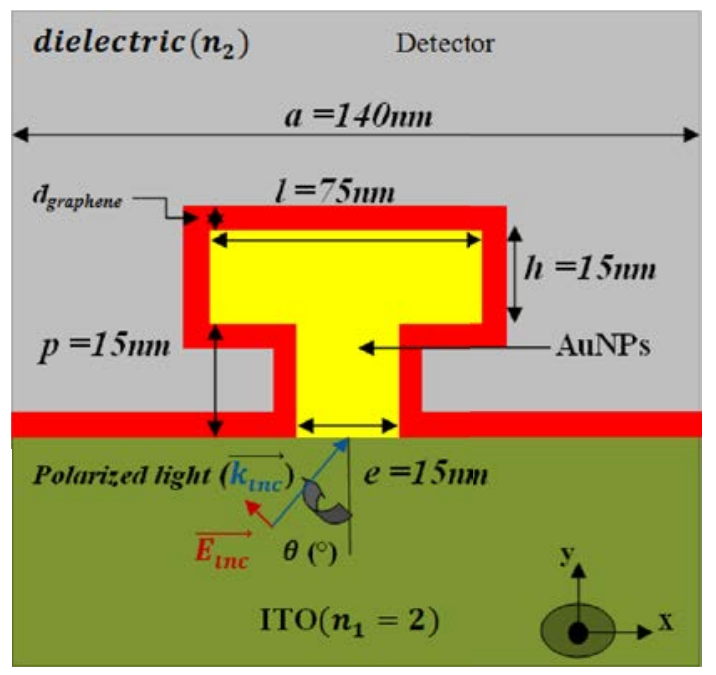

Fig. 1. Schematic representation of the structure studied in this work ITO/ AuNPs/graphene/dielectric, the gold nanoparticles are characterized $\mathrm{h}=15 \mathrm{~nm}, \mathrm{l}=75 \mathrm{~nm}, \mathrm{e}=15 \mathrm{~nm}$ and $\mathrm{p}=15 \mathrm{~nm}$, the mesh parameter $\mathrm{a}=140 \mathrm{~nm}$ is defined as the nearest distance between two nanoparticles of neighboring gold. The input source is placed in the substrate (ITO) and the detector in the air. deposited on AuNPs (ITO/AuNPs(form T)/graphene). The corresponding geometry is well illustrated in Fig. 1. This work is organized as follows: Section 2 briefly presents the theoretical model of the simulation and the applied method for calculating the sensitivity of the sensor as well as the absorption coefficient and the factor of merit. In section 3, the design representation of the proposed LSPR biosensor will be given. Section 4 will give the corresponding results for the case where AuNPs are coated with a graphene layer.

\section{Theoretical methods}

\subsection{Simulation model}

The optical properties of gold nanoparticles are determined numerically within a wavelength domain ranging from visible to near IR, specifically, [400 nm-1200 nm]. Simulations are performed using Finite Element Method (FEM) [37,38]. Actually, the simulation method has already been well documented in Refs. [39-41]; it consists in solving Maxwell's equations in the frequency domain by discretizing space in small finite elements (having various possible shapes such as triangular, mapped, tetrahedral (3D) etc.). Our calculation is carried out in a twodimensional (2D) domain with Ox and Oy axes oriented as shown in Fig. 1. An incident plane wave is generated within ITO substrate of refraction index $n_{1}=2$, and is launched along the Oy axis (direction of propagation). The plane wave is p-polarized (TM electromagnetic field) TM polarized and its associated electric field vibrates along the Ox axis. The calculation is meant to simulate an infinite array of nanoparticles along Ox axis, and the structure is supposed to be infinite along the direction of Oz. The space discretization is realized by means of free triangular mesh and such that the maximum size of elements is adjusted depending on the considered material. In all dielectrics (layer on top of AuNPs and ITO substrate), the elements size is no more than $\left(\frac{\lambda_{0}}{2 n}\right) / 5$, where $\mathrm{n}$ is the index of ITO or air/water. In gold particles the skin depth $\delta$ effect has to be taken into account and, as such, at least five points should be set across $\delta$. Within the considered frequency range, $\delta$ lies above $22 \mathrm{~nm}$ (following the calculation method of [42]), so a maximum elements size of about $3 \mathrm{~nm}$ is sufficient for the numerical calculations to converge. Actually, given that we use (in our structure) thin layers of graphene (from $0 \mathrm{~nm}$ to $5 \mathrm{~nm}$ ) in which light attenuates as well (being a transition metal class material), we decided to fix the maximum elements size at $6.10^{-3} \mathrm{~nm}$ (to achieve numerical convergence). Obviously, the mesh is relaxed everywhere else as explained above (in dielectric materials).

In Fig. 1, is sketched the simulation models domains in the considered configuration, that is, when nanoparticles are covered with thin layer of graphene (Fig. 1). The frequency-dependent complex permittivity of gold metal is described by the Lorentz-Drude model $[43,44]$ :

$\varepsilon(\omega)=\varepsilon_{r, \infty}+\sum_{m=0}^{M} \frac{f_{m} \omega_{p}^{2}}{\omega_{m}^{2}-\omega^{2}+j \omega \Gamma_{m}}$

Where $\varepsilon_{r, \infty}$ is the relative permittivity at infinite frequency, $\omega_{p}$ the plasma frequency, and $\omega_{m}, f_{m}$ and $\Gamma_{m}$ are the resonance frequency, the strength (weight) and the damping rate of the $m^{\text {th }}$ oscillator, respectively. The Lorentz-Drude model uses $\mathrm{M}$ damped harmonic oscillators to describe the small resonances observed in the metal's frequency response. The value of the dielectric constant at infinite frequency $\left(\varepsilon_{r, \infty}\right.$ $=1$ ) and all the other parameters of L-D model are taken from Ref. [43]. The formers are summarized in Table 1 below:

\subsection{Determination of the sensitivity}

The sensitivity of the refraction index is determined by the following equation [45]: 
Table 1

Gold ( $\mathrm{Au}$ ) Lorentz-Drude model parameters.

\begin{tabular}{lllll}
\hline Term & $f_{m}[\mathrm{rad} / \mathrm{s}]$ & $\omega_{p}[\mathrm{rad} / \mathrm{s}]$ & $\omega_{m}[\mathrm{rad} / \mathrm{s}]$ & $\Gamma_{m}[\mathrm{rad} / \mathrm{s}]$ \\
\hline $\mathrm{m}=0$ & 0.760 & $13.7210^{15}$ & 0.00 & $0.0805210^{15}$ \\
$\mathrm{~m}=1$ & 0.024 & $13.7210^{15}$ & $0.630510^{15}$ & $0.366110^{15}$ \\
$\mathrm{~m}=2$ & 0.010 & $13.7210^{15}$ & $1.26110^{15}$ & $0.524110^{15}$ \\
$\mathrm{~m}=3$ & 0.071 & $13.7210^{15}$ & $4.51110^{15}$ & $1.32210^{15}$ \\
$\mathrm{~m}=4$ & 0.601 & $13.7210^{15}$ & $6.53810^{15}$ & $3.78910^{15}$ \\
$\mathrm{~m}=5$ & 4.384 & $13.7210^{15}$ & $20.2410^{15}$ & $3.36410^{15}$ \\
\hline
\end{tabular}

$S=\frac{\Delta \lambda_{L S P R}}{\Delta n_{2}}\left(n m R I U^{-1}\right)$

Where $\mathrm{n}_{2}$ is the refraction index of the detection medium and $\lambda_{L S P R}$ is the resonance wavelength of localized surface plasmon resonance (LSPR) of gold nanoparticles (AuNPs). The detection medium could be either water $\left(n_{2}=1.333\right)$ or air $\left(n_{2}=1.000\right)$ materials $\left(\Delta n_{2}=0.333\right)$. The sensitivity of the structure arises from the change in refractive index induced by an adsorbate on the interface between the detection medium and the dielectric on top of AuNPs. However, after the point of saturation, a red-shift of plasmonic resonance of $\lambda_{L S P R}$ resonance has been observed.

\subsection{Calculation of absorption}

In the ATR configuration, according to the principle of energy conversation, the sum of absorption (A), Reflection (R) and transmission ( $\mathrm{T}$ ) shall be equal to 1 (i.e., $\mathrm{A}+\mathrm{R}+\mathrm{T}=1$ ) Assuming that there is no energy loss in addition to the absorption of Materials. Under the condition of ATR, (R) is always close to zero, hence the absorption of the system can be reduced to A = 1-R-T. When the LSPR was excited, the absorption gradually increased until it reached a maximum. The maximum absorption (A) was close to 1 indicating that the incident energy was almost completely absorbed by the stratified materials. Therefore, the maximum incident light energy transfer to the evanescent wave is necessary in order to achieve the best performance of LSPR improvement [46-49].

\subsection{Design consideration of proposed for the sensitive detection of sensors}

Fig. 1 each layer of materials is supposed to be stacked along the Oy axis. In our nanostructure, ITO is chosen as the refractive index coupling substrate $\mathrm{n}_{1}=2$, which deposited the gold nanoparticles and coated with a layer of graphene. The graphene layer $\left(\mathrm{d}_{\text {graphene }}\right)$ is close to the AuNPs (form T), and the other side of the graphene layer also connects with a non-absorbent $\mathrm{n}_{2}$ index Dielectric (sensing medium). The graphene layer is used as a biomolecular recognition element. The

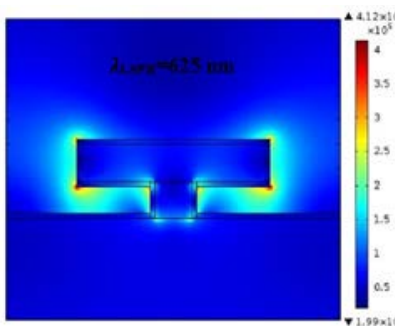

(a)
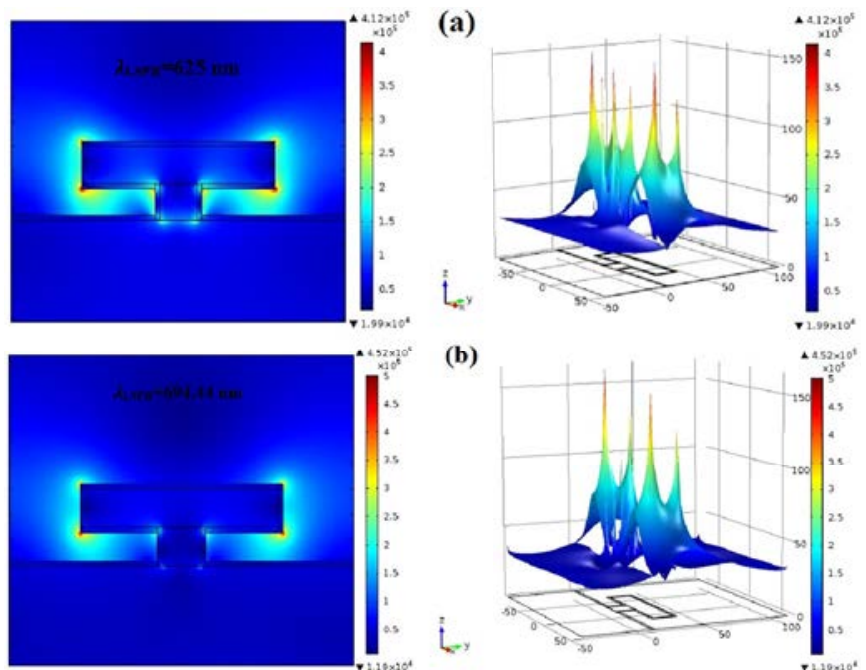

(b)
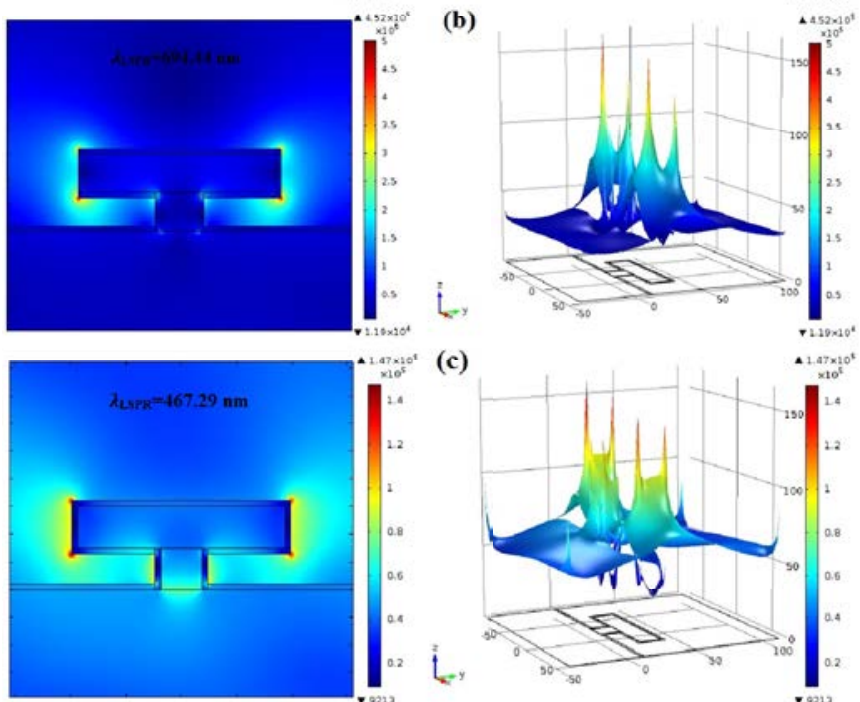

(c)

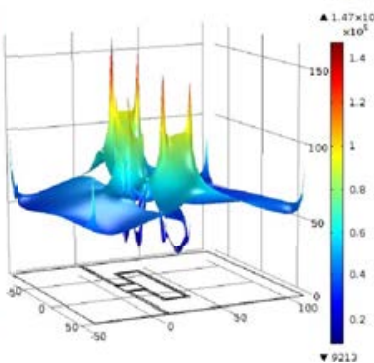

Fig. 3. Electric field strength distributions calculated by numerical analysis, for the normal incidence $(\theta=0)$ at the wavelength of the maximum absorption $625 \mathrm{~nm}(\mathrm{a})$, for the oblique incident $(\theta=80 \mathrm{deg})$ at the wavelength of the maximum absorption $694.44 \mathrm{~nm}$ mode (1) (b) and $467.29 \mathrm{~nm}$ mode (2) (c), of the structure studied ITO/AuNPs/graphene/Air, for $\mathrm{d}_{\text {graphene }}=2 \mathrm{~nm}$ and the gold nanoparticles are characterized by $e=15 \mathrm{~nm}, \mathrm{p}=15 \mathrm{~nm}, \mathrm{l}=75 \mathrm{~nm}$, $\mathrm{h}=15 \mathrm{~nm}$ and $\mathrm{a}=140 \mathrm{~nm}$. The refractive index of the substrate on which they are deposited is $\mathrm{n}_{1}=2$.

geometric parameters of the AuNPs were fixed as the diameter $\mathrm{l}=75 \mathrm{~nm}$, the height $\mathrm{h}=15 \mathrm{~nm}$, the interparticle distance $\mathrm{a}=140 \mathrm{~nm}$ and the thickness $\mathrm{p}=15 \mathrm{~nm}$ and the width $\mathrm{e}=15 \mathrm{~nm}$ of the base forming the "T". The complex permittivity of gold as a function of frequency is described by the Lorentz-Drude model Eq. (1).

The graphene deposited between the AuNPs and the detection medium to increase the macromolecular absorption capacity of the biosensor (fixation of the biological element). The thickness of the graphene is varied as $\mathrm{d}_{\text {graphene }}=\mathrm{L} * 0.34 \mathrm{~nm}$, where $\mathrm{L}$ is the number of
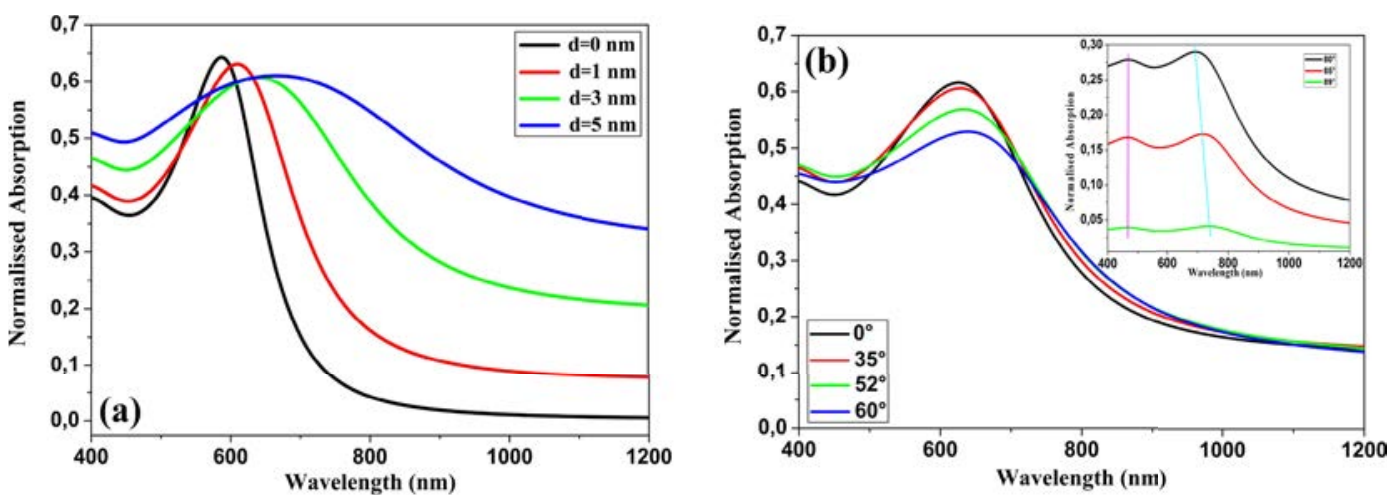

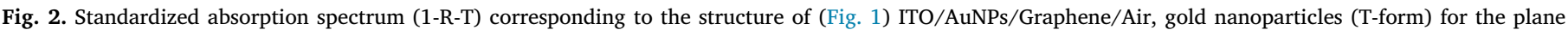

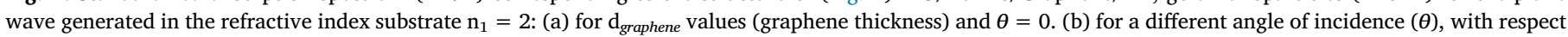

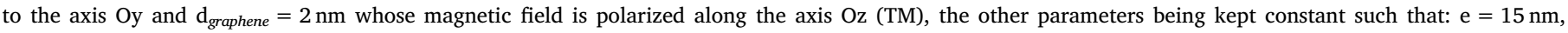
$\mathrm{p}=15 \mathrm{~nm}, \mathrm{l}=75 \mathrm{~nm}, \mathrm{~h}=15 \mathrm{~nm}$ and $\mathrm{a}=140 \mathrm{~nm}$. 

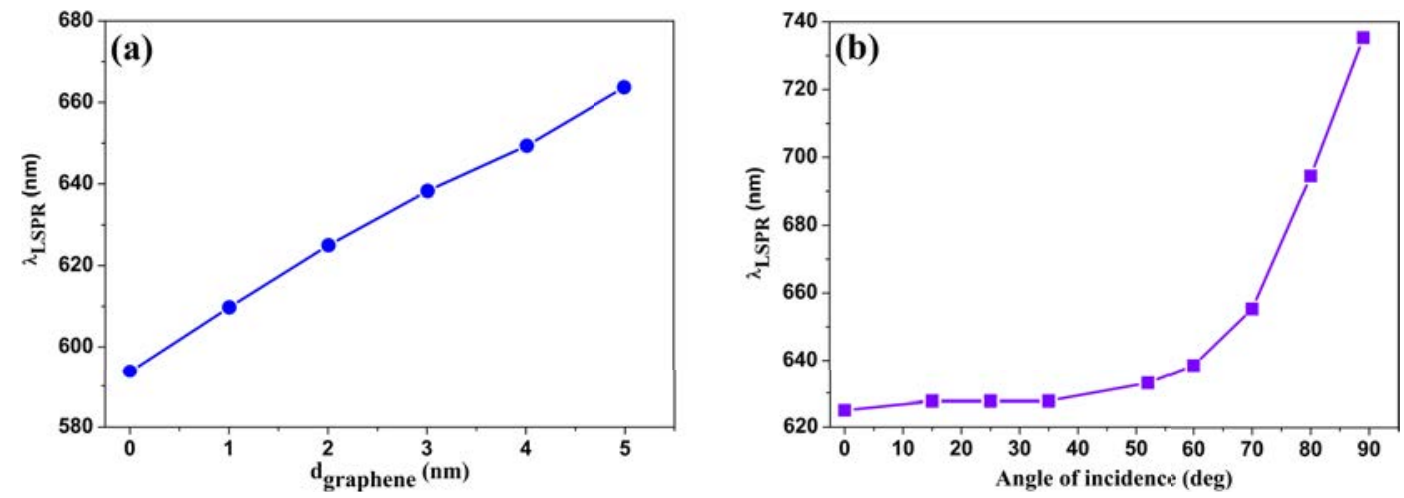

Fig. 4. Evolution of the resonance wavelength of the network ITO/AuNPs/graphene/Air, (a) with change of thickness of the ( $\left.d_{\text {graphene }}\right)$ graphene layer, (b) According to the angle of incidence $(\theta)$ relative to the axis Oy, the thickness of graphene covering the gold nanoparticles is $\mathrm{d}_{\text {graphene }}=2 \mathrm{~nm}$. For the planar wave generated in the refractive index substrate $\mathrm{n}_{1}=2$, whose magnetic field is polarized (TM) according to the Oz axis.
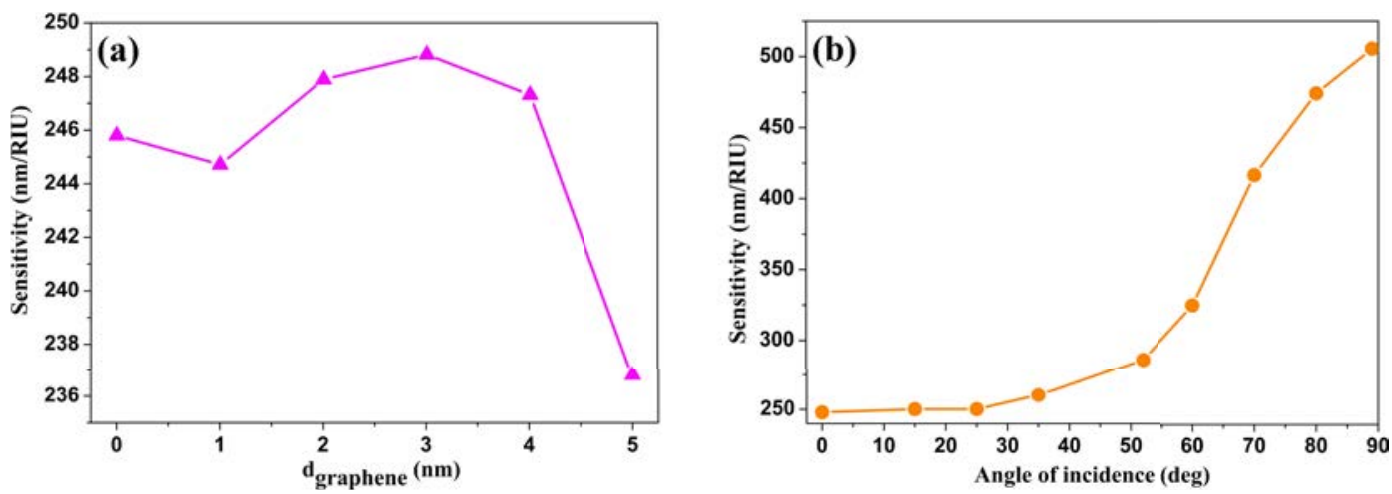

Fig. 5. Evolution of the system's sensitivity ITO/AuNPs/graphene/Air: (a) depending on the thickness of graphene covering the gold nanoparticles, (b) According to the angle of incidence $(\theta)$, relative to the axis Oy for $\mathrm{d}_{\text {graphene }}=2 \mathrm{~nm}$ and the nanoparticles are characterized by e $=15 \mathrm{~nm}, \mathrm{p}=15 \mathrm{~nm}, 1=75 \mathrm{~nm}, \mathrm{~h}=15 \mathrm{~nm}$ and $\mathrm{a}=140 \mathrm{~nm}$. The refractive index of the substrate on which they are deposited is $\mathrm{n}_{1}=2$.

graphene layers and its complex refractive index $\left(\mathrm{n}_{g}\right)$ in the visible range is an absorbent and dispersing material, the refractive index of which is given by the following Eq. (3) [50]:

$n_{g}=3.0+\frac{C_{1}}{3} \lambda$

Where the constant $C_{1}=5.446 \mu \mathrm{m}^{-1}$ [51], and is $\lambda$ the wavelength of the incident light in $\mu \mathrm{m}$. The refractive index of the detection layer is chosen as $\mathrm{n}_{2}=1.000$ or 1.333 .

\section{Results and discussion}

\subsection{Detection by wavelength interrogation of plasmonic resonance}

Examining, first, the nanoparticles (AuNPs) illustrated in Fig. 1. The evolution of absorption specters associated with FEM is indicated in Fig. 2. The function of wavelength $(\lambda)$ for polarization p (TM) (incident magnetic field similar the substrate interface) The cross section of the absorption of the plasmon resonance mode decreases with $\theta$ for the TM polarization, due to the fact that the incident field, transmitted through the ITO-Air interface, becomes perpendicular to the substrate at the critical angle of incidence $\beta=\operatorname{arsin}\left(n_{1} \sin (\theta) / n_{2}\right)$ and cannot be paired with horizontal mode.

However, the incident field is always parallel to the interface in the $\mathrm{TM}$ polarization, and is proportional to the transmission coefficient that increases near $\beta$, for this reason; the absorption of the plasmon resonance mode in plan increases the TM polarization with the angle of incidence $\theta$ of the plane wave [52,53].

In this paper, we investigated the detection potential of a $2 \mathrm{D}$ structured network of nanoparticles, plasmon reading, based on
Kretschmann configuration. Through a comprehensive study based on the FEM method, we presented the response of the structured surface to the fixation of a graphene layer. The comparison between the theoretical performance of the structured biosensors to that of conventional biosensors based on a purely propagative plasmon, leads to the importance of nanostructure in improving the sensitivity not only in relation to the response of a biosensor without a graphene but also in relation to the effect of micro-structuring of the surface which generates only a loss of performance. We found that the dominance of LSPR modes can lead to an improvement of a factor of $1.23 \%$ in terms of sensitivity to a deposit of a layer of graphene, while being less sensitive to variations of the graphene layer with the angle of incidence $\theta$ of the wave and leading to a gain of $103.90 \%$ in terms of sensitivity and $181.77 \%$ for FoM.

The absorption spectra of nanostructure are calculated in Fig. 2. The absorption is calculated for the normal incidence of changes in the thickness of the graphene layer covers the nanoparticles. As can be seen in Fig. 2(a), an increase in the thickness of the graphene film typically results in a red shift in the resonance lines due to the dielectric charge of the resonators.

Under the polarized-magnetic (TM) light at the angle of incident $\theta$, the nanoparticles network has two types of resonances Fig. 2(b): (i) a large resonance peak (mode 2) centered around $\lambda_{L S P R}=465 \mathrm{~nm}$ in the independent absorption spectrum of the angle of incidence; and (ii) a narrow resonance characteristic offset from $\lambda_{L S P R}=625 \mathrm{~nm}$ at $735 \mathrm{~nm}$ when the angle of incidence increased from $\theta=0$ to 89 (deg).

A near-field optical image is required to understand the physics behind the spectral distribution characteristics of the field associated with plasmon mode in nanostructure. Fig. 3 presents a comparison of the field properties simulations in two cases of normal and oblique 

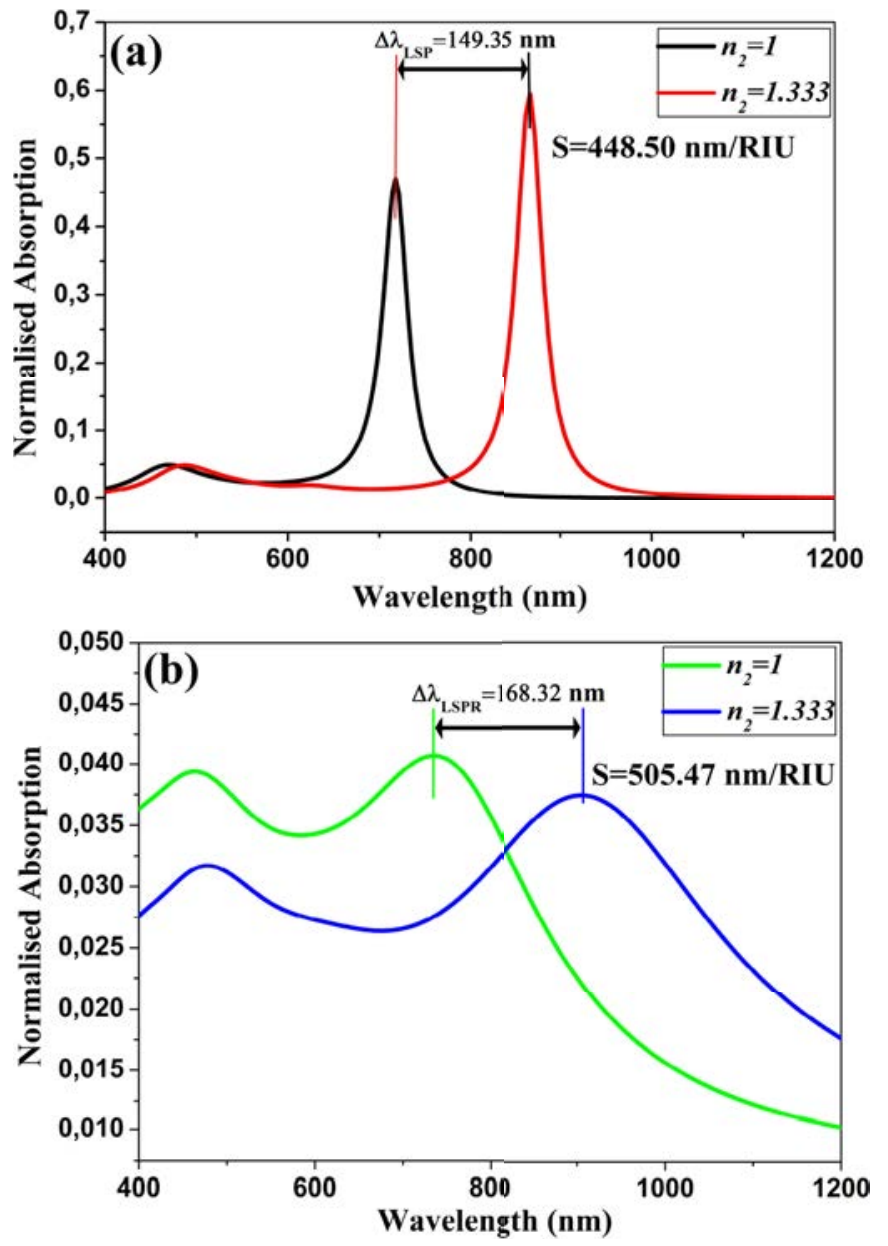

Fig. 6. Absorption as a function of wavelength incidence at incident angle 89 deg. (a) for configuration without a graphene layer and (b) for configuration with the graphene layer $(2 \mathrm{~nm})$.

Table 2

FoM Evolution of the system ITO/AuNPs/graphene, depending on the angle of incidence $(\theta)$ relative to the Oy axis. For $\mathrm{d}_{\text {graphene }}=2 \mathrm{~nm}$ and nanoparticles are characterized by $\mathrm{e}=15 \mathrm{~nm}, \mathrm{p}=15 \mathrm{~nm}, \mathrm{e}=75 \mathrm{~nm}, \mathrm{e}=15 \mathrm{~nm}$ and $\mathrm{a}=140 \mathrm{~nm}$. The refractive index of the substrate on which they are deposited is $\mathrm{n}_{1}=2$.

\begin{tabular}{lll}
\hline Angle of incidence (deg) & Mode1-FoM $\left(\mathrm{RIU}^{-1}\right)$ & Mode2-FoM $\left(\mathrm{RIU}^{-1}\right)$ \\
\hline 0 & 1.63 & - \\
15 & 1.67 & - \\
25 & 1.64 & - \\
35 & 1.77 & - \\
52 & 1.91 & - \\
60 & 2.20 & - \\
70 & 3.06 & - \\
80 & 4.60 & 0.42 \\
85 & 4.44 & 0.39 \\
89 & 4.23 & 0.34 \\
\hline
\end{tabular}

incidence. The structure is expected to change the sensitivity of the refractive index from the lower to the outside, since the confined field around nanoparticle [5,54].

The electric field distribution in the structure excited by the normal incidence $(\theta=0)$ is shown in Fig. 3(a). For the ITO/AuNPs/plasmon structure resonance, the field is mainly confined to the corners of the nanoparticle shape $(\mathrm{T})$ horizontal bar between the graphene layer and the sensing medium. In contrast, the structure at an excitation by a plane wave oblique $(\theta=80 \mathrm{deg})$ mode 2 by input to the axis (Oy)
Fig. 3(c). The configuration shows, however the field penetration is fore in the left and right sides of the horizontal bar, and smaller fraction of the field is confined to the areas exposed to the AuNPs post region $[5,10]$. By an excitation of an oblique plane wave $(\theta=80 \mathrm{deg})$ mode 1 , shows a field strength located at the corners of the horizontal bar only Fig. 3(b). Fig. 4 supports this assignment of spectral characteristics, because the large resonances in the plane are the same under transversely-magnetic (TM) polarized light excitation. The plasmon modes excited by the field components are sensitive to changes in the graphene layer thickness $\left(\mathrm{d}_{\text {graphene }}\right)$ and angle of incidence $(\theta)$.

The angular responses of the LSPR resonance are shown in Fig. 4(b), as well as the spectral response to thicknesses of the variable graphene layer Fig. 4(a). It is seen that for the surface of ITO/AuNPs/graphene/ Air, is satisfied for the angle up to $89 \mathrm{deg}$. In these curves we present the comparison of the wavelength evolution of plasmon resonance of nanonetwork of gold form nanoparticles (T) dependent on the angle of incidence and thicknesses of the layer of graphene of different optical properties of the field associated with the plasmon polarizes magnetic Transverse (TM) mode. The measured wavelength evolution of the absorption lattice resonance according to $\mathrm{d}_{\text {graphene }}$ corresponds extremely well with the calculated values and trends. As linearly increases from $\lambda_{L S P R}=573.82 \mathrm{~nm}$ to $\lambda_{L S P R}=663.71 \mathrm{~nm}$ Fig. 4(a) when the thickness of the graphene layer increases from 0 to $5 \mathrm{~nm}$, rogue LSPR increases rapidly.

Which is shown in Fig. 5(a), the curve shows a dramatic increase in sensitivity with the thickness of graphene layer varying from 1 to $3 \mathrm{~nm}$, and the greater sensitivity is $248.82 \mathrm{~nm} / \mathrm{RIU}$ can be obtained at $\mathrm{d}_{\text {graphene }}=3 \mathrm{~nm}$. After this, the sensitivity starts to decrease rapidly up to $236.81 \mathrm{~nm} / \mathrm{RIU}$ at $\mathrm{d}_{\text {graphene }}=5 \mathrm{~nm}$ [5].

Sensitivity is closely related to the change in plasmon resonance wavelength (LSPR). When the largest LSPR is obtained at $\theta=89 \mathrm{deg}$ and $\mathrm{d}_{\text {graphene }}=2 \mathrm{~nm}$, the corresponding sensitivity also approaches to the maximum. The relationship between the sensitivity and the angle of incidence represented by the curve in Fig. 5(b), we notice a variation of sensitivity very slowly up to $35 \mathrm{deg}$ and then converges quickly. Significantly, the suppression of radiative decomposition of extended resonance lifetime in the two-dimensional lattice of nanoparticles form (T) can be accomplished without needing grid orders to pass from radiative to evanescent. From it, we can clearly determine that the maximum sensitivity as high as $505.47 \mathrm{~nm} / \mathrm{RIU}$ can be relative to angle 89 (deg). When comparing with the configuration without a graphene layer $\left(\mathrm{d}_{\text {graphene }}=0\right)$ the sensitivity is $448.50 \mathrm{~nm} / \mathrm{RIU}$ as shown in Fig. 6 , the sensitivity may have a significant improvement of $12.70 \%$ with the graphene layer of $2 \mathrm{~nm}$.

The numerical computed sensitivities are used for estimating the resonance offset by Eq. (2) and are given in Fig. 5. The results are obtained for resonance excitation and for the offset of the detection medium refractive index $\left(\Delta n_{2}=0.333\right)$. The offsets are compared with the direct computation of the resonance frequencies using the lattice without a graphene layer and with the graphene layer, at least 103.90 $\%$ of the sensitivity enhancement is observed.

The change in FoM for the proposed biosensor is shown in Table 2. When the detection medium refractive index varies from 1 to 1.333 , the width change to the range of $F W H M=152.11 \mathrm{~nm}$ to FWHM $=103.22 \mathrm{~nm}$, then we can calculate the FoM by the formula: FoM $=\mathrm{S}$ / FWHM. The result shows that the FoM first increases from $1.63 \mathrm{RIU}^{-1}$ to $4.59 \mathrm{RIU}^{-1}$ when varies from 0 to $80 \mathrm{deg}$, then decreased with the angle of incidence of the plane wave of $4.59 \mathrm{RIU}^{-1}$ to $4.23 \mathrm{RIU}^{-1}$ when varies from 80 to $89 \mathrm{deg}$ for plasmon resonance mode (1) [55,56]. For the calculated FoM of Mode (2) appeared from the angle incidence 80 deg remains low and has near constant $0.40 \mathrm{RIU}^{-1}$.

We can expect that the structure having an exposed surface of the graphene layer has an improved sensitivity and FoM to the variation of the angle of incidence. To understand these trends in detail, we will focus on the variations of geometric parameters of the bi-dimensional nanostructures of nanoparticles of gold form (T), which shows the 

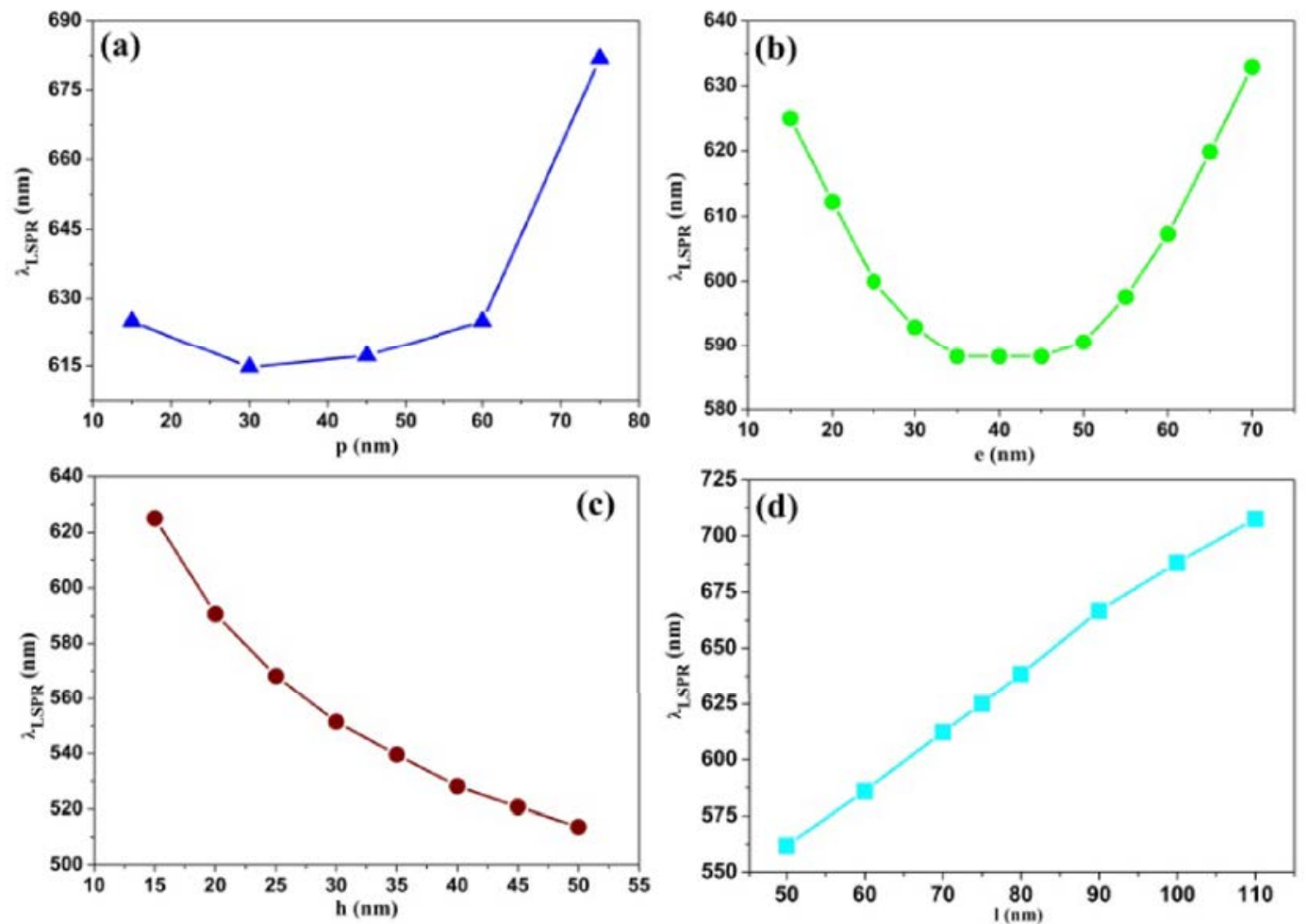

Fig. 7. Evolution of the LSPR peak of ITO/AuNPs/graphene/Air system, according to geometric parameters of gold nanoparticles: (a) and (b) the height $\mathbf{p}$ and width e of the vertical nanoparticle bar (form T), (c) and (d) the height $\mathbf{h}$ and width $\mathbf{1}$ of the bar Horizontal nanoparticle (form T). The nanoparticles are characterized by, $\mathrm{p}=15 \mathrm{~nm}, \mathrm{l}=75 \mathrm{~nm}, \mathrm{~h}=15 \mathrm{~nm}$ and $\mathrm{a}=140 \mathrm{~nm}$ and covered by $\mathrm{d}_{\text {graphene }}=2 \mathrm{~nm}$. The refractive index of the substrate on which they are deposited is $\mathrm{n}_{1}=2$.
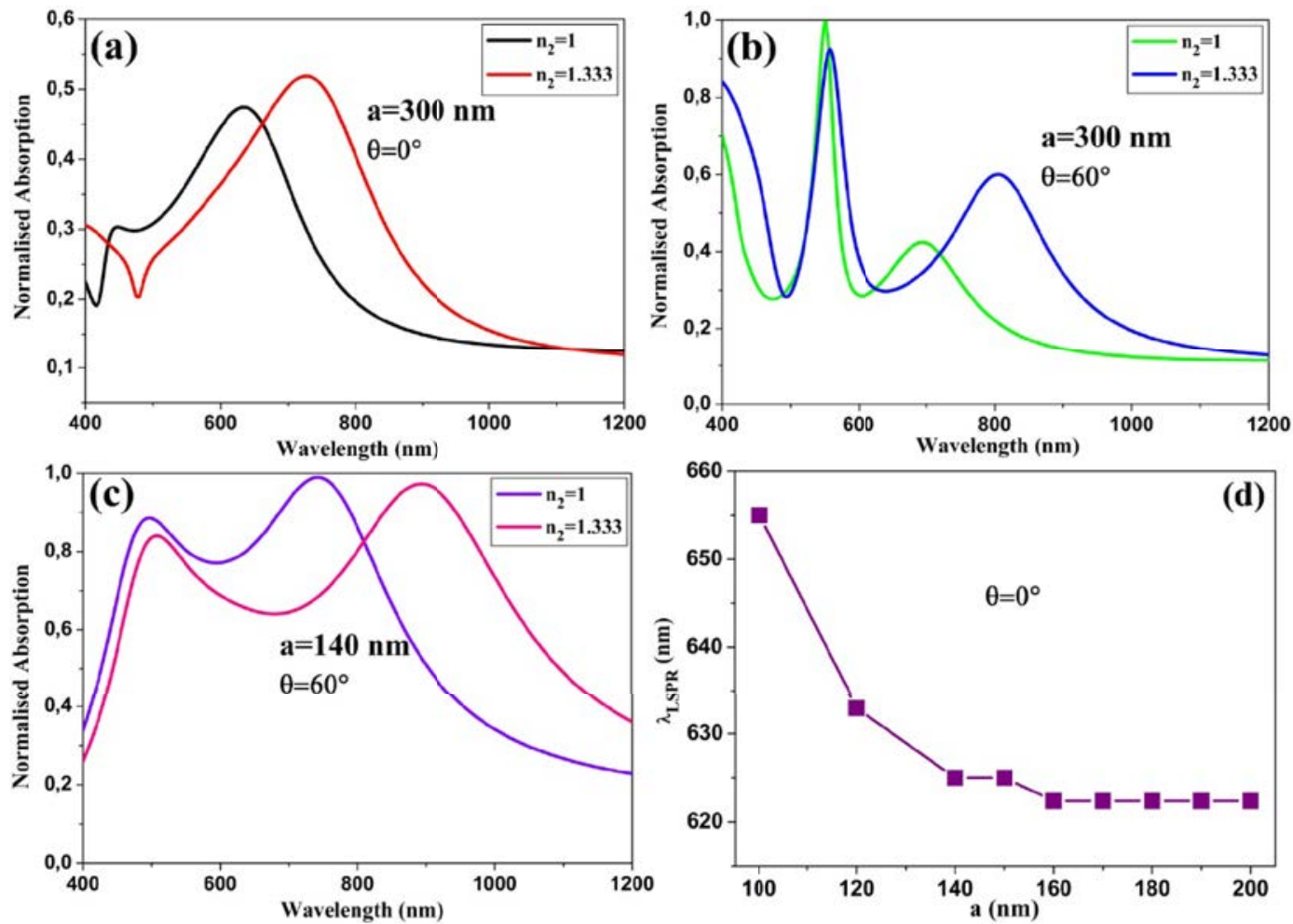

Fig. 8. Change in absorption from the incident wavelength for a LSPR ITO/AuNPs/Graphene/Air biosensor for different network period: (a) for a $=300 \mathrm{~nm}$ at normal incidence $\theta=0$, (b) for $\mathrm{a}=300 \mathrm{~nm}$ at Oblique incidence $\theta=60 \mathrm{deg}$, (c) for $\mathrm{a}=140 \mathrm{~nm}$ at The normal incidence $\theta=0$ and (d) evolution of the system peak LSPR, depending on the period "a" to $\theta=0$. 

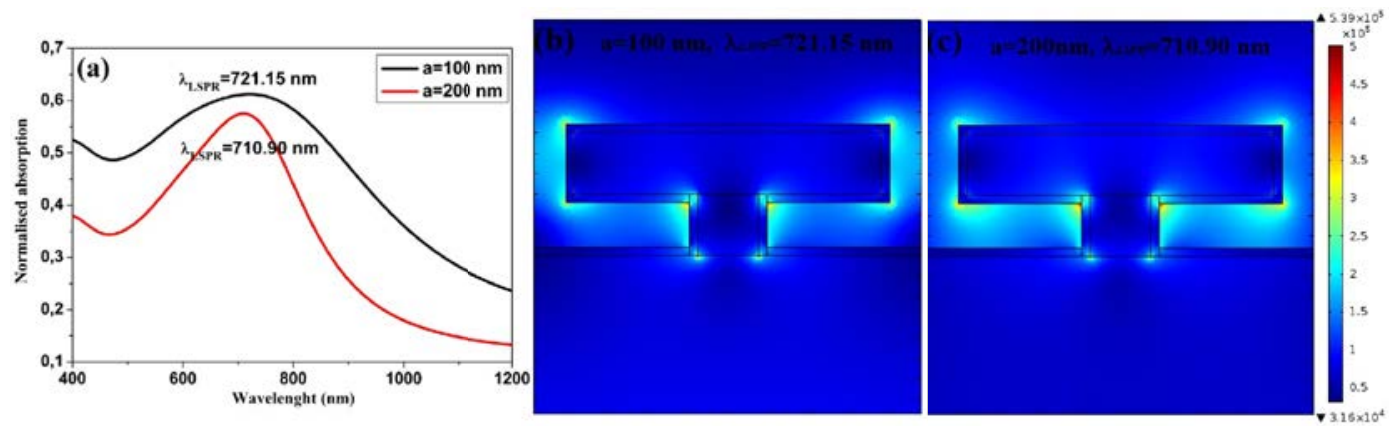

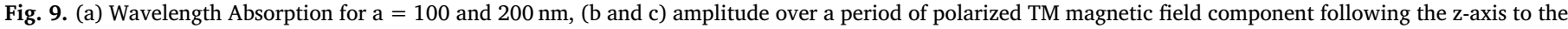
system resonance described in Fig. 1 at $\lambda_{L S P R}=721.15 \mathrm{~nm}$ and $\lambda_{L S P R}=710.90 \mathrm{~nm}$ respective, for the detection medium $\mathrm{n}_{2}=1.333$.

plasmon resonances of network surface [54].

Fig. 7 represents the results of the various numerical simulations of the evolution of optical responses under the polarized light TM as normal incidence. As expected, one of the parameters is varied and fixed the other parameters of nanoparticles, to obtain the resonance wavelength of the spectral peak LSPR.

Under the transverse-magnetic (TM) polarized light at the normal incidence, the height $(\mathrm{p})$ of the vertical bar of nanoparticles presents a shift of the LSPR resonance Fig. 7(a), a narrow resonance characteristic that moved from $\lambda_{L S P R}=615 \mathrm{~nm}-682 \mathrm{~nm}$ when the height (p) of the nanoparticles increased from $\mathrm{p}=30 \mathrm{~nm}-75 \mathrm{~nm}$. The height of the vertical bar (p) depending on the optical response suggests that the spectral feature can be associated with a shift to the red wavelength. The electric field excites the plasmon modes in planar lattices that depend strongly on the height (p) of the nanoparticles. While if one varies the width of the vertical bar (e) of nanoparticles Fig. 7(b) note shift to the blue after to the wave-languor red, when the width (e) varies from 15 to $35 \mathrm{~nm} \lambda_{L S P R}$ passes from $625 \mathrm{~nm}$ to $588 \mathrm{~nm}$ after having the plasmon resonance wavelength remains noting at $\lambda_{L S P R}=588 \mathrm{~nm}$ for the values of $\lambda_{L S P R}=588 \mathrm{~nm}$ for the values of $\mathrm{e}=35,40$ and $45 \mathrm{~nm}$, then if we follow to increase the width (e) from 45 to $70 \mathrm{~nm}$ one gets important shift of resonance $\lambda_{L S P R}=633 \mathrm{~nm}$ this attribution supports the spectral characteristics, because the resonance is strongly sensitive to changes in width (e).

It is observed in Fig. 7(c) that the plasmon resonance wavelength decreases when (h) increases. In fact, it goes from $625 \mathrm{~nm}$ for $\mathrm{h}=15 \mathrm{~nm}-514 \mathrm{~nm}$ for $\mathrm{h}=50 \mathrm{~nm}$. In other words, the height of the nanoparticles results in a decrease of $111 \mathrm{~nm}$ when (h) increases by $35 \mathrm{~nm}$. As for the width (1) of the nanoparticles Fig. 7(d), the larger it is, the better the sensitivity. This is a $\lambda_{L S P R}$ of $562 \mathrm{~nm}$ for $1=50 \mathrm{~nm}$ at a $\lambda_{L S P R}$ of $691 \mathrm{~nm}$ for $1=120 \mathrm{~nm}$, an increase in the resonance wavelength of about $129 \mathrm{~nm}$ for an increased width of $70 \mathrm{~nm}$.

Finally, it is noted that the sensitivity increases when the period (a) of the network decreases and the impact nail increases. The variation in sensitivity according to the period parameter (a) was not carried out for the structure considered here. Commented in the first part of this work illustrating the influence of the nail impact on the sensitivity Fig. 5(b) when (a) is fixed, that increasing period generates a loss of sensitivity. For example, the network of gold nanoparticles in contact with a layer of graphene $(2 \mathrm{~nm})$ under the sensing medium, deposited on a refractive index substrate $\mathrm{n}_{1}=2$, and characterized by the parameters $1=75 \mathrm{~nm}$, $\mathrm{h}=15 \mathrm{~nm}, \mathrm{e}=15 \mathrm{~nm}, \mathrm{p}=15 \mathrm{~nm}$, at the normal incidence $(\theta=0)$ and $\mathrm{a}=300 \mathrm{~nm}$ had a sensitivity of $278 \mathrm{~nm} / \mathrm{RIU}$ for $\mathrm{a}=300 \mathrm{~nm}$ and $\theta=60 \mathrm{deg}$ a sensitivity of $336.38 \mathrm{~nm} / \mathrm{RIU}$, an increase of $21 \%$ Fig. 8(a) and (b). For $\theta=60 \mathrm{deg}$ and $\mathrm{a}=140 \mathrm{~nm}$ had a sensitivity of $451.30 \mathrm{~nm} / \mathrm{RIU}$ for $\mathrm{a}=300 \mathrm{~nm}$ and $336.38 \mathrm{~nm} / \mathrm{RIU}$, an increase of $34.16 \%$ when the period decreased by $160 \mathrm{~nm}$ Fig. 8(b) and (c).

The period of the network studied is related to the interparticle distance by the relationship (a-1). Therefore, at fixed width $(1=75 \mathrm{~nm})$, the smaller the period, the more the nanoparticles will be close to each other. Thus, studying the influence of (a) on the plasmon response is tantamount to studying the interparticle coupling to the resonance.

Fig. 9 shows the evolution of LSPR according to the period of nanoparticles. One can separate the curve into two parts:

First, below a $=100$ at $160 \mathrm{~nm}$, the decrease of $\lambda_{L S P R}$ with the increase of the period becomes abruptly very large, $\lambda_{L S P R}=655 \mathrm{~nm}$ for $\mathrm{a}=100 \mathrm{~nm}$ and $\lambda_{L S P R}=622.40 \mathrm{~nm}$ for $\mathrm{a}=160 \mathrm{~nm} \lambda_{L S P R}$ decreases by $32.6 \mathrm{~nm}$ when going from $\mathrm{a}=100 \mathrm{~nm}$ to $\mathrm{a}=160 \mathrm{~nm}$. In fact, when the period goes from 160 to $200 \mathrm{~nm}, \lambda_{L S P R}$ remains constant at $622.40 \mathrm{~nm}$ $[5,15]$. As we have just seen in the previous paragraph, this type of behavior is characterized as a coupling between nanoparticles via their evanescent fields [29]. This shift to $\lambda_{L S P R}$ Blue is also accompanied by an increase in sensitivity ( $\mathrm{S}=198.60 \mathrm{~nm} / \mathrm{RIU}$ for $\mathrm{a}=100 \mathrm{~nm}$ and $\mathrm{S}=265.75 \mathrm{~nm} / \mathrm{RIU}$ for $\mathrm{a}=200 \mathrm{~nm}$ ).

Fig. 9 shows the amplitude of the magnetic field component following the z-axis, to the resonance of the system described in Fig. 9(a) $\lambda_{L S P R}=721.15 \mathrm{~nm}$ for $\mathrm{a}=100 \mathrm{~nm}$ and $\lambda_{L S P R}=710.90 \mathrm{~nm}$ for $\mathrm{a}=200 \mathrm{~nm}$ ). As with the previous field mapping (Fig. 3), we see that the area where the field is most intense is located at the corners formed by the two " $\mathrm{T}$ " riser bars. It is therefore highly probable that the sensitivity increases again if it is arranged that the dielectric covering the nanoparticles $\left(\mathrm{n}_{g}\right)$ also follows their relief in these areas, thus releasing a new access to the detection medium $\left(\mathrm{n}_{2}\right)$.

\section{Conclusion}

In this study, we proposed a new LSPR biosensor to achieve high sensitivity. In the proposed biosensor of gold form nanoparticles (T), the layers of graphene are used to increase the sensitivity, even as the graphene is applied as the element of biomolecular recognition. The projective of the biosensor is sensitive to the change in the refractive index of the detection medium. We examined the influence of the angle of incidence on the sensitivity of the biosensor. The result shows that a relatively high sensitivity and FoM can be obtained when the index angle is relatively increased. To obtain a high sensitivity, we apply $2 \mathrm{~nm}$ of graphene in the proposed structure, which means that the biosensor was very sensitive to the wave incidence oblique with the substrate surface, $505.47 \mathrm{~nm} / \mathrm{RIU}$ for sensitivity and $4.59 \mathrm{RIU}^{-1}$ for the factor of Merit. In addition, we have shown that an appropriate number of the graphene layers can generate a positive impact to improve the maximum sensitivity as high as $248.82 \mathrm{~nm} / \mathrm{RIU}$ can be obtained with three layers of graphene coated on the nanoparticles at the normal incidence. Finally, we showed that the sensitivity can be improved about $103.90 \%$ and the FoM improved about $181.77 \%$.

\section{Appendix A. Supplementary data}

Supplementary data to this article can be found online at https:// doi.org/10.1016/j.physe.2019.02.010. 


\section{References}

[1] R.W. Wood, Philos, XLII. On a remarkable case of uneven distribution of light in a diffraction grating spectrum, Mag 4 (21) (1902) 396-402.

[2] U. Fano, The theory of anomalous diffraction gratings and of quasi-stationary waves on metallic surfaces (Sommerfeld's waves), J. Opt. Soc. Am. 31 (3) (1941) 213.

[3] A. Hessel, A.A. Oliner, A new theory of Wood's anomalies on optical gratings, Appl. Opt. 4 (10) (1965) 1275.

[4] A.G. Nikitin, T. Nguyen, H. Dallaporta, Narrow plasmon resonances in diffractive arrays of gold nanoparticles in asymmetric environment: Experimental studies, Appl. Phys. Lett. 102 (2013) 221-116.

[5] M. El Barghouti, A. Akjouj, A. Mir, Effect of graphene layer on the localized surface plasmon resonance (LSPR) and the sensitivity in periodic nanostructure, Photon. Nanostruct. Fundam. Appl. 31 (2018) 107-114.

[6] A. Otto, Excitation of nonradiative surface plasma waves in silver by method of frustrated total reflection, Z. Phys. 216 (4) (1968) 398-410.

[7] E. Kretschmann, H. Raether, Notizen: radiative decay of nonradiative surface plasmons excited by light, Z. Naturforsch. A 23 (12) (1968) 2135-2136.

[8] E. Hao, G.C. Schatz, Electromagnetic fields around silver nanoparticles and dimers, J. Chem. Phys. 120 (2004) 357.

[9] S. Zou, G.C. Shatz, Silver nanoparticle array structures that produce giant enhancements in electromagnetic fields, Chem. Phys. Lett. 403 (2005) 62.

[10] M. El Barghouti, A. Akjouj, A. Mir, Numerical analysis of $\mathrm{MoS}_{2}$ on the LSPR in periodical nanostructure of $\mathrm{Ag}$ arrays/graphene for sensitive sensors, Int. J. Microwave Opt. Technol. 13 (2018) 454-461.

[11] M.D. Malinsky, K.L. Kelly, G.C. Schatz, R.P. Van Duyne, Chain length dependence and sensing capabilities of the localized surface plasmon resonance of silver nanoparticles chemically modified with alkanethiol self-assembled monolayers, J. Am. Chem. Soc. 123 (2001) 1471-1482.

[12] J. Zhao, L. Jensen, J. Sung, S. Zou, G.C. Schatz, R.P. Van Duyne, Interaction of plasmon and molecular resonances for rhodamine $6 \mathrm{G}$ adsorbed on silver nanoparticles, J. Am. Chem. Soc. 129 (2007) 7647-7656.

[13] J.N. Anker, W. Paige Hall, O. Lyandres, N.C. Shah, J. Zhao, R.P. Van Duyne, Biosensing with plasmonic nanosensors, Nat. Mater. 7 (2008) 442-453.

[14] E.M. Larsson, J. Alegret, M. Kall, D.S. Sutherland, Sensing characteristics of NIR localized surface plasmon resonances in gold nanorings for application as ultrasensitive biosensors, Nano Lett. 7 (2007) 1256-1263.

[15] M. El Barghouti, A. Akjouj, A. Mir, Effect of $\mathrm{MoS}_{2}$ layer on the LSPR in periodic nanostructures, Optik 171 (2018) 237-246.

[16] E. Galopin, A. Noual, J.N. Jonsson, J.N. Martin, A. Akjouj, Y. Pennec, B.D. Rouhani, R. Boukherroub, S. Szunerits, Short- and long-range sensing using plasmonic nanostructures: experimental and theoretical studies, J. Phys. Chem. C113 (2009) $15921-15927$.

[17] L. Touahir, E. Galopin, R. Boukherroub, A.C. Gouget-Laemmel, J.N. Chazalviel, F. Ozanam, O. Saison, A. Akjouj, Y. Pennec, B. Djafari-Rouhani, S. Szunerits, Plasmonic properties of silver nanostructures coated with an amorphoussiliconcarbon alloy and their applications for sensitive sensing of DNA hybridization, J. Phys.: Analyst 136 (2011) 1859.

[18] L. Wu, H.S. Chu, W.S. Koh, E.P. Li, Highly sensitive graphene biosensors based on surface plasmon resonance, Optic Express 18 (2010) 14395-1440.

[19] C. Lertvachirapaiboon, A. Baba, S. Ekgasit, C. Thammacharoen, K. Shinbo, K. Kato, F. Kaneko, Gold nanoparticles synthesis used for sensor applications, IEEE ConfProc ISEIM. INSPEC Accession Number, 2014 1435-1199.

[20] C. Cen, J. Chen, C. Liang, J. Huang, X. Chen, Y. Tang, Z. Yi, X. Xu, Y. Yi, S. Xiao, Plasmonic absorption characteristics based on dumbbell-shaped graphene metamaterial arrays, Phys. E Low-dimens. Syst. Nanostruct. 103 (2018) 93-98.

[21] S.H. Choi, Y.L. Kim, K.M. Byun, Graphene-on-silver substrates for sensitive surface plasmon resonance imaging biosensors, Optic Express 19 (2011) 458-466.

[22] T. Maurer, R. Nicolas, G. Lévêque, P. Subramanian, J. Proust, J. Béal, S. Schuermans, J.-P. Vilcot, Z. Herro, M. Kazan, J. Plain, R. Boukherroub, A. Akjouj, B. Djafari-Rouhani, P.-M. Adam, S. Szunerits, Enhancing LSPR sensitivity of Au gratings through graphene coupling to Au film, Plasmonics 9 (2014) 507-512.

[23] R. Nicolas, G. Lévêque, P.M. Adam, T. Maurer, Graphene doping induced tunability of nanoparticles plasmonic resonances, Plasmonics 13 (2018) 1219-1225.

[24] G. Giovannetti, P.A. Khomyakov, G. Brocks, V.M. Karpan, J. van den Brink, P.J. Kelly, Doping graphene with metal contacts, Phys. Rev. Lett. 101 (2008) 026803.

[25] Z. Fang, Y. Wang, Z. Liu, A. Schlather, P.M. Ajayan, F.H.L. Koppens, P. Nordlander, N.J. Halas, Plasmon-induced doping of graphene, ACS Nano 6 (2012) $10222-10228$

[26] J. Wu, C. Zhou, J. Yu, H. Cao, S. Li, W. Jia, Design of infrared surface plasmon resonance sensors based on graphene ribbon arrays, Optic Laser. Technol. 59 (2014) 99-103.

[27] A.H. Castro Neto, F. Guinea, N.M.R. Peres, K.S. Novoselov, A.K. Geim, The electronic properties of graphene, Rev. Mod. Phys. 81 (1) (2009) 109-162.

[28] L. Wu, H.S. Chu, W.S. Koh, E.P. Li, Highly sensitive graphene biosensors based on surface plasmon resonance, Optic Express 18 (14) (2010) 14395-14400.

[29] M. El Barghouti, A. Mir, A. Akjouj, Sabine Szunerits, Modulation of localized surface plasmon resonances of a silver nanoparticle array upon the presence of MoS2 coatings or underlying thin films, Optik 179 (2019) 819-827.
[30] T.-Y. Chang, M. Huang, A.A. Yanik, H.-Y. Tsai, P. Shi, S. Aksu, M.F. Yanik, H. Altug, Large-scale plasmonic microarrays for label-free high-throughput screening, Lab Chip 11 (2011) 3596.

[31] O. Saison-Francioso, G. Lévêque, A. Akjouj, Y. Pennec, B. Djafari-Rouhani, R. Boukherroub, S. Szunerits, Search of extremely sensitive near-infrared plasmonic interfaces: a theoretical study, Plasmonics (2013) 1-8, https://doi.org/10.1007/ s11468-013-9588-9.

[32] E. Galopin, et al., Sensitivity of plasmonic nanostructures coated with thin oxide films for refractive index sensing: experimental and theoretical investigations, J. Phys. Chem. C 114 (27) (2010) 11769-11775.

[33] K.S. Novoselov, A.K. Geim, S.V. Morozov, D. Jiang, Y. Zhang, S.V. Dubonos, I.V. Grigorieva, A.A. Firsov, Electric field effect in atomically thin carbon films, Science 306 (2004) 666-669.

[34] R.R. Nair, P. Blake, A.N. Grigorenko, K.S. Novoselov, T.J. Booth, T. Stauber, N.M.R. Peres, A.K. Geim, Fine structure constant defines visual transparency of graphene, Science 320 (2008) 1308-1308.

[35] C. Sun, X.Q. Wang, Y.X. Zheng, T.H. Yang, M.J. Zeng, Plasmonic tuning in mid infrared regime with a composite array of graphene ribbons and silver nanowires, Phys. E Low-dimens. Syst. Nanostruct. 97 (2018) 384-391.

[36] D.C. Elias, R.V. Gorbachev, A.S. Mayorov, S.V. Morozov, A.A. Zhukov, P. Blake, L.A. Ponomarenko, I.V. Grigorieva, K.S. Novoselov, F. Guinea, A.K. Geim, Dirac cones reshaped by interaction effects in suspended graphene, Nat. Phys. 7 (2011) 701-704.

[37] D.Y. Lei, A.I. Fernández-Domínguez, Y. Sonnefraud, K. Appavoo, R.F. Haglund, J.B. Pendry, S.A. Maier, Revealing plasmonic gap modes in particle-on-film systems using dark-field spectroscopy, ACS Nano 6 (2012) 1380-1386.

[38] A. Noual, Y. Pennec, A. Akjouj, B. Djafari-Rouhani, L. Dobrzynski, Nanoscale plasmon waveguide including cavity resonator, J. Phys. Condens. Matter 21 (2009) 375301.

[39] M.W. Knight, N.J. Halas, Nanoshells to nanoeggs to nanocups: optical properties of reduced symmetry core-shell nanoparticles beyond the quasistatic limit, New J. Phys. 10 (2008) 105006.

[40] A.M. Gilbertson, Y. Francescato, T. Roschuk, V. Shautsova, Y. Chen, T.P.H. Sidiropoulos, M. Hong, V. Giannini, S.A. Maier, L.F. Cohen, R.F. Oulton, Plasmon-induced optical anisotropy in hybrid graphene-metal nanoparticle systems, Nano Lett. 15 (2015) 3458-3464.

[41] Y. Zhan, D.Y. Lei, X. Li, S.A. Maier, PlasmonicFano resonances in nanohole quadrumers for ultra-sensitive refractive index sensing, Nanoscale 6 (2014) 4705-4715.

[42] R. Qiang, R.L. Chen, J. Chen, Modeling electrical properties of gold films at infrared frequency using FDTD method, Int. J. Infrared Millim. Waves 25 (2004) 1263.

[43] A.D. Rakic, A.B. Djuristic, J.M. Elazar, M.L. Majewski, Analysis of optical channel cross talk for free-space optical interconnects in the presence of higher-order transverse modes, Appl. Opt. 37 (1998) 5271-5283.

[44] C.J. Powell, Analysis of optical- and inelastic-electron-scattering data. II. Application to Al, J. Opt. Soc. Am. 60 (1970) 78-93.

[45] E. Galopin, J. Niedziołka-Jonsson, A. Akjouj, Y. Pennec, B. Djafari-Rouhani, A. Noual, R. Boukherroub, S. Szunerits, Sensitivity of plasmonic nanostructures coated with thin oxide films for refractive index sensing: experimental and theoretical investigations, J. Phys. Chem. C 114 (2010) 11769-11775.

[46] S. Zeng, et al., A review on functionalized gold nanoparticles for biosensing applications, Plasmonics 6 (2011) 491-506.

[47] R. Verma, B.D. Gupta, R. Jha, Sensitivity enhancement of a surface plasmon resonance based biomolecules sensor usinggraphene and silicon layers, Sensor. Actuator. B Chem. 160 (2011) 623-631.

[48] J. Homola, S.S. Yee, G. Gauglitz, Surface plasmon resonance sensors: review, Sensor. Actuator. B Chem. 54 (1999) 3-15.

[49] A. Lahav, M. Auslender, I. Abdulhalim, Sensitivity enhancement of guided-wave surface-plasmon resonance sensors, Opt. Lett. 33 (2008) 2539-2541.

[50] M. Bruna, S. Borini, Optical constants of graphene layers in the visible range, Appl. Phys. Lett. 94 (3) (2009) Art. no. 213116.

[51] Z. Fang, Y. Wang, Z. Liu, A. Schlather, P.M. Ajayan, F.H.L. Koppens, P. Nordlander, N.J. Halas, Plasmon-induced doping of graphene, ACS Nano 6 (11) (2012) 10222-10228.

[52] A.M. Gilbertson, Y. Francescato, T. Roschuk, V. Shautsova, Y. Chen, T.P.H. Sidiropoulos, M. Hong, V. Giannini, S.A. Maier, L.F. Cohen, R.F. Oulton, Plasmon-induced optical anisotropy in hybrid graphene-metal nanoparticle systems, J. Nano. Letters 15 (2015) 3458-3464.

[53] N. Mahi, G. Lévéque, O. Saison, J.M. Djouda, R. Caputo, A. Gontier, T. Maurer, P.M. Adam, B. Bouhafs, A. Akjouj, In depth investigation of lattice plasmon modes in substrate-supported gratings of metal monomers and dimers, J. Phys. Chem. C 121 (2017) 2388-2401.

[54] Y. Kaya, S. Ayas, A.E. Topal, H. Guner, A. Dana, Sensitivity comparison of localized plasmon resonance structures and prism coupler, Sensor. Actuator. B Chem. 191 (2014) 516-521.

[55] L. Wu, Y. Jia, L. Jiang, J. Guo, X. Dai, Y. Xiang, D. Fan, Sensitivity improved SPR biosensor based on the $\mathrm{MoS}_{2}$ /graphene-aluminum hybrid structure, J. Lightwave Technol. 35 (1) (2017) 82-87.

[56] L. Wu, J. Guo, X. Dai, Y. Xiang, D. Fan, Sensitivity enhanced by $\mathrm{MoS}_{2}$-graphene hybrid structure in guided-wave surface plasmon resonance biosensor, J. Plasmonics (2017) 1-5. 